\title{
Statistical optimization of gelatin immobilisation on modified surface PCL microcarrier to improve PCL microcarrier compatibility
}

\begin{abstract}
Growing cells on microcarriers may have overcome the limitation of conventional cell culture system. However, the main challenge remains at ensuring the surface biocompatibility with cells. Polycaprolactone (PCL), a biodegradable polymer, has received considerable attention because of its excellent mechanical properties and degradation kinetics that suit various applications, but its non-polar hydrocarbon moiety renders it sub-optimal for cell attachment. In this present study, the aim was to improve biocompatibility of PCL microcarrier by introducing oxygen functional group via ultraviolet irradiation and ozone aeration $\left(\mathrm{UV} / \mathrm{O}_{3}\right.$ system) to allow covalent immobilization of gelatin on the PCL microcarrier surface. Respond surface methodology was used as a statistical approach to optimized parameters that effect the immobilization of gelatin. The parameters used to maximized amount of gelatin immobilize were the mol ratio of COOH:EDAC, NHS concentration and gelatin concentration. The optimum conditions for maximum amount of gelatin $(1797.33 \mu \mathrm{g} / \mathrm{g})$ on the surface of PCL were as follows: 1.5 of $\mathrm{COOH}$ :EDAC ratio, $10 \mathrm{mM}$ NHS concentration and, $80 \mathrm{mg} / \mathrm{ml}$ gelatin. The result shows that gelatin coated PCL microcarrier promote more and rapid cell adhesion with density of as compared to raw PCL microcarrier ( and $\mathrm{UV} / \mathrm{O}_{3}$ treated PCL microcarrier ( . Therefore, immobilization of gelatin with optimized parameters onto PCL microcarrier improved biocompatibility of PCL microcarrier.
\end{abstract}

Keyword: Microcarrier; Gelatin immobilization; Polycaprolactone; Statistical optimization 
